\title{
An Extreme Learning Machine-based Pedestrian Detection Method
}

Kai Yang, Eliza Y. Du, Edward J.Delp, Pingge Jiang, Feng Jiang, Yaobin Chen, Rini Sherony, and Hiroyuki Takahashi

\begin{abstract}
Pedestrian detection is a challenging task due to the high variance of pedestrians and fast changing background, especially for a single in-car camera system. Traditional HOG+SVM methods have two challenges: (1) false positives and (2) processing speed. In this paper, a new pedestrian detection method using multimodal HOG for pedestrian feature extraction and kernel based Extreme Learning Machine (ELM) for classification is presented. The experimental results using our naturalistic driving dataset show that the proposed method outperforms the traditional HOG+SVM method in both recognition accuracy and processing speed.
\end{abstract}

